Four patients noted tinnitus after four to 16 weeks of therapy. One of these four developed nerve deafness after 12 weeks; hearing was absent in a frequency range above 1000 cycles/s. Within 10 weeks of stopping the drug the hearing deficit recovered to the pretreatment range of hearing. In this patient the nerve deafness recurred on re-starting therapy and recovered again when the drug was again discontinued. Although other side effects in other patients were reported, such as nausea and vomiting, there was no report of optic atrophy.

Schirrmacher ${ }^{5}$ reported a case where a 35 year old woman with anaemia and chronic nephritis was treated with $100 \mathrm{mg}$ of cobalt chloride daily for six months, by which time she had developed many adverse effects of treatment including bilateral nerve deafness. Audiometry showed a decrease in sound perception in all frequencies and caloric tests were also abnormal. Five months later, after stopping the drug, her hearing had improved. There was no optic atrophy.

Even though our patient's hearing deficit has improved considerably since his initial presentation, 11 months after stopping work he still has a residual auditory dysfunction. According to the literature cobalt toxicity (as a result of cobalt treatment) results in transient nerve deafness with full recovery. Our patient may have had some hearing impairment before cobalt exposure as he himself feels that his hearing is almost back to normal.

The high blood levels of cobalt and definite improvement in vision and hearing occurring within one month of stopping work, strongly suggests that cobalt toxicity was the cause of the deafness and visual loss. It has been reported to the Health and Safety Executive. No other case has been identified to our knowledge.

We are grateful to Mr P K Wishart for his ophthalmological help, and to Mr G Lightfoot, for the neuro-otological assessment. Also to Dr A Taylor, Robens Institute, Guildford, Surrey, for measurement of the cobalt levels.

HEATHER M MEECHAM PETER HUMPHREY Department of Neurosciences,

Walton Hospital, Rice Lane, Liverpool L9 1 AE, UK

1 Payne LR. The hazards of cobalt. J Soc Occup Med 1977;27:20-25.

2 Gardner FH. The use of Cobaltous Chloride in Anaemia associated with Chronic Renal Disease. J Lab Clin Med 1953;41:56-63.

3 Editorial. Cobalt in severe renal failure. The Lancet 1976:2;26-27.

4 Licht A, Oliver M, Rachmilewitz EA. Optic Atrophy following treatment with Cobal Chloride in a patient with pancytopenia and hypercellular marrow. Israeli $J$ Med $S c i$ 1972;8:61-65.

5 Schirrmacher UOE. Case of cobalt poisoning. BMJ 1967;1:544-5.

Bilateral perineuritis of the optic nerves

Perineuritis of the optic nerves is a rare inflammatory condition which presents a typical clinical picture of recurrent episodes of visual loss associated with pain and neuroradiological features resembling an optic nerve sheath meningioma. It has become increasingly recognised and is an important differential diagnosis of optic nerve meningioma as it is potentially treatable. We report a case and emphasise the clinical and neuroradiological features that allow its differentiation from other lesions of the optic nerves.

In 1985 a 31 year old Greek woman presented with three stereotyped episodes of reduced vision in the left eye over an eighteen month period. Her right eye was normal and she had no other neurological deficit. Two weeks before each attack she had experienced facial pain. The visual loss occurred over several days and was associated with pain on ocular movements. The attacks were treated with short courses of systemic steroids and each time the vision returned to almost normal over several weeks. We first examined the patient at the third episode. Right visual acuity was $6 / 5$ and left counting fingers. On the left there was colour loss to all Ishihara plates, pain on ocular movement and disc pallor. There was no intraocular inflammation or proptosis. The right field was normal, the left showed only preservation of a rim of peripheral field in the upper nasal quadrant of the left eye.

Flash VERs showed normal responses in the right eye but delay and low amplitude with an abnormal wave form in the left eye. Responses to pattern reversal were absent in the left eye but showed normal latency and slightly reduced amplitude in the right. A diagnosis of left optic neuritis was made.

The patient returned home when her vision was back to normal but following another attack a few months later there was no visual recovery in her left eye. Soon after this she had an attack in the right eye. Over the next 18 months she had three further episodes in the right eye each preceded by bilateral frontal headache and pain in the face two weeks before. She had no pain on ocular movement or on retropulsion of the globe and although her vision improved after each of these episodes, it never recovered to its previous level.

On review in 1987 the right visual acuity was $6 / 12$ with reduced colour perception, the left eye had no perception of light. There was left optic atrophy and temporal pallor of the right disc. Perimetry showed a superior temporal defect in the right eye and a diagnosis of a chiasmal lesion was made. General and neurological examination was normal apart from a previous valvotomy for mitral stenosis. Routine haematology including haemoglobin electrophoresis, biochemistry, protein electrophoresis, autoantibody screen (ANA + ve 1:10), serum angiotensin converting enzyme, chest, sinus and skull radio-

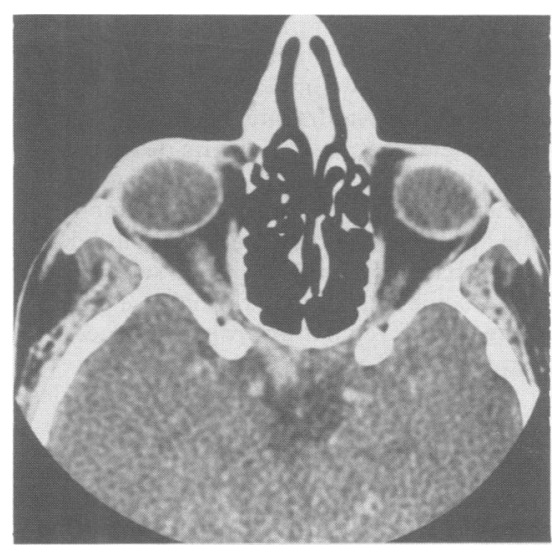

Figure $1 C T$ scan (axial scan) showing bilateral thickening of both optic nerves with ragged outlines. graphs were all normal. Circulating immune complexes were raised (PEG method $7.8 \mathrm{mg}$ IgG/dl, normal <4.9). Ear, nose and throat consultation was unremarkable. A lumbar puncture showed normal cells, protein and no oligoclonal band. CT scan (fig 1) showed bilateral thickening and ragged outlines of the optic nerves with enlargement of the chiasm. MRI confirmed these findings. A diagnosis of an intrinsic inflammatory lesion of the optic nerves and chiasm of unknown aetiology was made and she was started on oral prednisolone, $60 \mathrm{mg}$ daily which was tailed off and discontinued over six months. At this time the neuro-ophthalmic signs were unchanged but CT scan appearances had improved.

She remained well without steroids for another seven months but then had a relapse in the right eye. An exploratory craniotomy was performed to obtain a tissue diagnosis. At surgery the right optic nerve appeared normal, the left grey and atrophic. There were no adhesions or evidence of tumour. The left optic nerve was excised for histology. This showed an optic nerve largely replaced by collagenous fibrous tissue. In places this was infiltrated with small numbers of lymphocytes and plasma cells (fig 2). In one area a few nerve fibres remained. Some of these were myelinated, others had lost their myelin sheaths. The patient was restarted on systemic steroids and two years later the vision remained stable with no further relapses.

The history of relapsing visual loss, associated with ipsilateral headache, pain on ocular movement and retropulsion of the globe, initially suggested demyelinating disease; later neuroradiological evidence of thickening of the optic nerves and sheaths was more typical of optic nerve sheath meningioma. ${ }^{1}$ The prominent history of steroid responsiveness and pain associated with the attacks, however, made an inflammatory lesion likely.

The features of an "inflammatory" optic nerve lesion and neuroradiological changes similar to optic nerve sheath meningioma, together with the pathological appearances of the optic nerve ${ }^{4}$ are compatible with perineuritis of the optic nerves. Dutton reported four patients with this condition ${ }^{1}$ and there have been a number of other case reports which emphasise the difficulties of differential diagnosis from optic neuritis or optic nerve sheath meningioma. ${ }^{2-7}$ Eight out of nine of these cases were female and ages ranged from 22-68 years at presentation, although most patients presented in the 40-55 age range. Pain was a noticeable feature in all except two cases. Visual loss has been reported to be

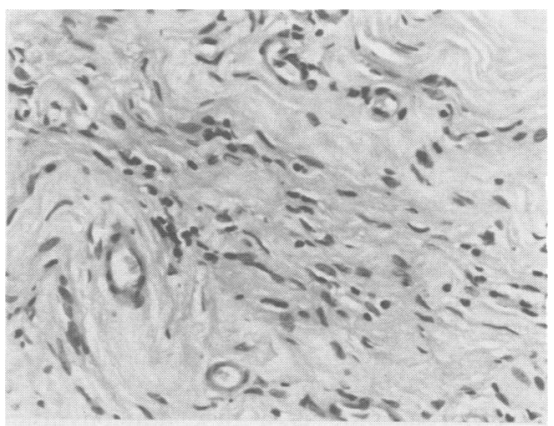

Figure 2 Section of left optic nerve showing extensive fibrosis and a sparse infiltrate of lymphocytes and plasma cells $(H+E$ $\times 400$ ). 
either relapsing or progressive over a period of six to 12 months by the time of presentation. Two patients have had bilateral disease. ${ }^{47}$ Some patients have responded to and stabilised on steroids and in a number, surgical biopsy has been performed to confirm the diagnosis. Dutton felt that surgical decompression of the nerve helped stabilise the condition.

Optic perineuritis is best confirmed histologically, because other causes of optic nerve sheath lesions, particularly meningioma cannot always be excluded by neuroimaging alone. Most reports have described thickening of the optic nerve sheath from fibrotic changes with varying amounts of chronic lymphocytic or plasma cell infiltrate or granulomatous changes. The intracranial nerve has been found to be pale and atrophic with chronic inflammatory infiltration as in our own case and that of Zhang ${ }^{2}$ or swollen with perivasculitis. ${ }^{3}$ The most complete description of pathological changes ${ }^{4}$ showed concentric deposition of collagenous fibroconnective tissue in the dural sheath with necrobiotic granulomas and a chronic inflammatory infiltrate causing a compressive optic neuropathy with ischaemic infarction. The inflammatory reaction did not extend beneath the pia mater. Electron microscopy showed exuberant fibroplasia, collagenosis and elastogenesis associated with focal extracellular collagen degeneration. The histological changes are non-specific. In suspected cases, biopsy is better taken from the intraorbital nerve rather than intracranially so that detailed examination of the optic nerve sheath can be performed.

$$
\begin{array}{r}
\text { PHILIP G HYKIN } \\
\text { DAVID J SPALTON } \\
\text { Medical Eye Unit, St Thomas' Hospital, } \\
\text { London SE1 7EH, UK }
\end{array}
$$

Correspondence to: $\mathrm{Mr}$ Spalton

1 Dutton JJ, Anderson RL. Idiopathic inflammatory perioptic neuritis simulating optic nerve sheath meningioma. Am J Ophthalmo 1985;100:424-30.

2 Zhang TL, Shao S, Zhang T, et al. Idiopathic inflammation of optic nerve simulating optic nerve sheath meningioma: CT Demonstration. J Comput Assist Tomogr 1987;11(ii): tion.

3 Rush JA, Kennerdell JS, Martinez J. Primary idiopathic inflammation of the optic nerve. $A m$ idiopathic inflammation of the

4 Margo CE, Levy MH, Beck RW. Bilateral idio CE, Levy MH, Beck RW. Bilateral idiopathic inflammation of the
sheaths. Ophthal 1989;96:200-6.

5 Krohel GB, Howard C, Smith RS. Granulomatous optic neuropathy. Arch Ophthal 1981;99:1053-5.

6 Kennerdell JS, Dresner SC. The non-specific orbital inflammatory syndromes. Surv Ophthal 1984;29:93-103.

7 Fering ER, Weatherbie L. Hypertrophic granulomatous cranial pachymeningitis causing blindness in a chronic dialysis patient. Neurol Neurosurg Psychiatry 1975;38:1170-6.

\section{Oxcarbazepine sensitivity treated by} desensitisation

Allergic reactions to anticonvulsant drugs may require a change of therapy. An alternative strategy is to desensitise the patient to the offending drug. This has already been described with carbamazepine. ${ }^{1}$

Oxcarbazepine is a new anticonvulsant drug developed as an alternative for patients unable to tolerate carbamazepine and supposedly causing fewer side-effects and allergic reactions. ${ }^{2}$ As yet, it is only available on a named-patient basis. Sensitivity to both carbamazepine and oxcarbazepine does occur. We describe a patient successfully managed by densensitisation to oxcarbazepine. We believe this is the first reported case of successful densensitisation to oxcarbazepine.

This 23 year old single man with mild mental handicap lives with his parents. He had a head injury in 1979 resulting in a posttraumatic amnesia of 24 hours. He developed complex partial seizures in 1982 , but treatment was not started until 1983 when he had his first generalised seizure. A resting EEG was then normal. Phenytoin greatly reduced his seizure frequency but resulted in problems of slowed cognition and mild toxicity.

In 1987 he developed a psychotic illness characterised by persecutory delusions, delusions of reference and third person derogatory auditory hallucinations. He described the hallucinations as occurring episodically. He had three admissions to a local psychiatric hospital over the next 18 months but the psychotic phenomena continued despite high-dose antipsychotic medication.

In view of his problems on phenytoin and the possibility that the hallucinations may have been related to epileptic phenomena rather than schizophrenia, he was started on carbamazepine in August 1988. Within one week he had developed a fever, generalised erythematous rash and lymphadenopathy. His leucocyte count was raised with an eosinophilia. All these symptoms and signs resolved over the next eight days after carbamazepine was withdrawn.

He was then referred to this hospital for further assessment. Continuous EEG monitoring for five days was unhelpful as he had no hallucinations during this time and the EEG remained normal. Oxcarbazepine was prescribed as an alternative to carbamazepine. Within 12 hours of the first dose $(300 \mathrm{mg})$ he developed a generalised itchy erythematous rash and a fever. Leucocyte count was within the normal range but with a mild eosinophilia. Oxcarbazepine was stopped and his symptoms subsided within 36 hours with antipyretic treatment alone.

Desensitisation was then attempted using low dose oxcarbazepine capsules prepared by the local pharmacy. Starting at $0 \cdot 1 \mathrm{mg}$ daily, the dose was doubled every two days. On day 2 , however, he developed mild itching and erythema on his hands and abdomen, but remained systemically well. The next dose increase was withheld until the symptoms disappeared 24 hours later. On day $19(50 \mathrm{mg} /$ day) he developed mild itching and erythema confined to his hands. Again the dosage increase was withheld until the symptoms subsided 144 hours later. No further adverse experiences were seen during desensitisation. By day 63 he achieved $1200 \mathrm{mg}$ per day. Phenytoin was then tailed off. One month later he was well established on this dose of oxcarbazepine, with no adverse effects, and with some reduction in the hallucinations. $\mathrm{He}$ remains on regular antipsychotic medication and lithium carbonate.

When allergic drug reactions occur, desensitisation can be a useful alternative to changing anticonvulsant therapy. In view of the risk of a severe drug reaction (blood dyscrasia, renal or hepatic toxicity) desensitisation should only be undertaken in selected cases where there are no other satisfactory alternatives. Oxcarbazepine is a promising new anticonvulsant and should not necessarily be abandoned when such sensitivity occurs, but a desensitisation regime considered instead.

DARRYL WATTS ONATHAN BIRD Burden Neurological Hospital,
Bristol BS16 1QT, UK

1 Smith $\mathrm{H}$, Newton $\mathrm{N}$. Adverse reactions to carbamazepine managed by desensitisation. Lancet 1985;i:753.

2 Editorial. Oxcarbazepine. Lancet 1989;ii:196-8.

Buspirone in the treatment of levodopa induced dyskinesias

Long-term levodopa treatment of patients with Parkinson's disease is commonly complicated by on-off fluctuations and dyskinesias. While recent advances have been made in the treatment of fluctuations ${ }^{1}$ inter-dose dyskinesias have become an increasing problem in the management of the long-term levodopa syndrome. Dopamine receptor antagonists have been shown to be effective in reducing levodopa induced dyskinesias but only at the expense of increased Parkinsonian disability. ${ }^{23}$ We have tested the azapirone drug buspirone which has mixed dopamine-2 receptor agonist-antagonist properties and main serotonin-1A agonist activity. ${ }^{4}$

Five patients (one female, four male) with idiopathic Parkinson's disease gave their informed consent to participate in the trial. Their mean age was $56 \cdot 2(47-72)$ years, mean duration of disease $10 \cdot 2(6-20)$ years and of levodopa treatment $8 \cdot 2(4-15)$ years. All had on-off fluctuations as well as disabling peakdose dyskinesias and all but one were treated with intermittent subcutaneous injections of apomorphine (mean daily dose $14 \mathrm{mg}$, range 4-30 mg) in addition to their oral levodopa (mean daily dose $865 \mathrm{mg}$, range 625$1.250 \mathrm{mg}$ ).

In three patients acute challenges with single doses of buspirone (10 and $20 \mathrm{mg}$ ) given 30 minutes before an apomorphine injection did not show any influence on the severity of involuntary movements (AIM scale) when compared with that seen after apomorphine alone. The "on" quality of motor response (Webster scale) was not affected and no side effects occurred.

All patients were then treated with daily doses of 15, 30, 45 and $60 \mathrm{mg}$ of buspirone over three consecutive days. For assessment of inter-dose dyskinesias patients kept a selfscoring diary for three days before starting buspirone and throughout the treatmen period. Involuntary movements were scored 1 when mild (not interfering with daily routine), 2 when moderate (interfering with daily routine, but able to continue) and 3 when severe (unable to continue with daily routine)

All five patients experienced a 10 to 40 (mean 20 ) $\%$ reduction of disabling (score 2 and 3) involuntary movements, three at $15 \mathrm{mg}$ of buspirone per day and one patient each at 30 and $60 \mathrm{mg}$, respectively. In three of them this was, however, associated with an increased frequency of "off" periods. Although compensated by an increased number of apomorphine injections without increasing dyskinesias in two patients this form of "titration" treatment was considered too complicated to embark on long-term therapy. In two patients the anti-dyskinetic daily dose 